\title{
Depression influences the quality of diabetes-related self-management activities in elderly patients with type 2 diabetes: a cross-sectional study
}

This article was published in the following Dove Press journal:

Clinical Interventions in Aging

26 April 2016

Number of times this article has been viewed

\section{Gabriela Mut-Vitcu' \\ Bogdan Timar ${ }^{2}$ \\ Romulus Timar' \\ Cristian Oancea ${ }^{3}$ \\ loan Cosmin $\mathrm{Citu}^{4}$}

'Second Department of Internal Medicine, ${ }^{2}$ Department of Functional Sciences, ${ }^{3}$ Department of Infectious

Diseases, ${ }^{4}$ Department of Obstetrics and Gynecology, "Victor Babes"

University of Medicine and Pharmacy,

Timisoara, Romania
Correspondence: Bogdan Timar Department of Functional Sciences, "Victor Babes" University of Medicine and Pharmacy, 2 Eftimie Murgu,

Timisoara 30004I, Romania

Tel +40 74I 528093

Fax +40256462856

Email timar.bogdan@umft.ro
Purpose: To evaluate the prevalence of depression and its impact on the quality of diabetes-related self-care activities in elderly patients with type 2 diabetes.

Patients and methods: In this cross-sectional study, 184 patients with type 2 diabetes were enrolled. Depression was evaluated using Patient Health Questionnaire-9 while the quality of diabetes-related self-care activities was assessed using the Summary of Diabetes-Related Self Care Activities Questionnaire.

Results: In our study group, $53.3 \%$ of the patients had moderate depression, $17.9 \%$ had severe depression, and $28.8 \%$ had no depression symptoms. Patient's age $(P=0.024)$, presence of diabetic neuropathy $(P<0.001)$, and body mass index $(P=0.037)$ proved to be independent and significant predictors for developing depression in patients with type 2 diabetes. The severity of depression was reverse correlated with the quality of self-care activities for all the studied components: global score $(r=-0.305)$, diet intervention score $(r=-0.297)$, exercise score $(r=-0.388)$, glycemic monitoring score $(r=-0.055)$, and feet care score $(r=-0.180)$. The presence of severe depression was associated with an increased prevalence of diabetes complications such as diabetic neuropathy and chronic kidney disease.

Conclusion: The prevalence of depression is higher in patients with type 2 diabetes compared to general population. Depression has a major negative impact on the quality of diabetes-related self-care activities and, being a treatable condition, proactive screening followed in case of a positive diagnosis by adequate treatment should be performed in all patients with diabetes.

Keywords: diabetes, depression, self-care, quality of life

\section{Introduction}

Obtaining a good glycemic control and, in the meantime, avoiding the therapeutic adverse events (eg, hypoglycemia) represent the main aim of the treatment of patients with diabetes, a challenge necessary in order to avoid the development of acute and chronic irreversible complications of diabetes mellitus (DM). The DM management measures include two major components: the health care professional's interventions and the patient's diabetes-related self-care interventions. Considering the particular attributes of DM, particularly being a chronic disease which is mainly treated in an outpatient setting, it is agreed that patient's self-management of the disease is of paramount importance in achieving the therapeutic goals while avoiding the therapeutic adverse events. ${ }^{1-3}$ The most cited diabetes-related self-care activities (DRSCA) include: blood glucose measurement with a consecutive adjustment of medication (eg, insulin dose) and amount of carbohydrates ingested during the adjacent meals, physical exercise interventions aiming mainly to lower the blood glucose levels and 
to diminish the insulin resistance-associated phenomenon, proactive screening for other diabetes complications and comorbidities emphasizing the importance of self-care, and inspection of feet, a site for frequent localization of diabetic ulcers. Diabetic ulcers are also observed in patients suffering from diabetic neuropathy (DN) and in many cases are undetectable without an appropriate visual inspection; these patients do not experience pain in the extremities in the advanced stages of neuropathy.

Emotional well-being is also an important part of diabetes care and self-management. Psychological and social problems can impair the individual's or family's ability to carry out diabetes care tasks and therefore compromise health status. ${ }^{4-7}$

Depression is a condition frequently associated with DM. The co-occurrence of depression and DM is emerging as a worldwide issue. Findings of epidemiological studies have shown a twofold increase in prevalence of depression and anxiety among patients with type 1 (T1DM) and type 2 DM (T2DM) compared with general population, adversely affecting the quality of life and DM-specific outcomes. ${ }^{4,-12}$ The cause and increased incidence of depression in DM patients remains poorly understood, the association between the two disorders being complex and bidirectional and might also share common biological determinants. ${ }^{13}$ Moreover, increased incidence of both DM and depression is associated with a more advanced age, emphasizing thus the association between the two conditions. ${ }^{8}$

Depression, in general, is cited to affect patient's adherence and compliance to treatment regardless of the disease. Considering the importance of these two components in the global management of diabetes, depression is a more significant predictor for a negative outcome. Findings of cross-sectional studies of the association between depression and diabetes self-care showed that healthy eating, regular exercise, and intake of low-calorie and low-fat food items showed a strong negative correlation with depression symptoms and diabetes distress, but not with the presence of clinical depression. ${ }^{14-18}$ This makes us think about the effect of differences between depressive symptoms and diagnosed depression on patient self-care and self-control. Clearly, the impact of depression symptoms and diabetes distress on self-care needs to be studied further. ${ }^{14}$

Considering this hypothesis, our study aims to evaluate the prevalence of depression in elderly patients with DM and to evaluate the impact of depression severity on the quality of DRSCA. A significant impact of depression on the quality of DRSCA would lead to possible new interventions aiming to improve the outcome of DM, mediated by both improvements in depression symptomatology and emphasizing the role of DRSCA interventions in patients with DM and advanced depression.

\section{Materials and methods Study design and participants}

For this cross-sectional, noninterventional study, we enrolled patients according to a population-based consecutive case principle. The study included 184 patients $(80$ men and 104 women), previously diagnosed with T2DM, attending scheduled visits at the Outpatient Clinic of the Emergency Hospital Timisoara between June 2014 and August 2015. All patients signed an informed consent form prior to performing any study procedure or measurement.

The study protocol was approved by the Ethics Committee of the Emergency Hospital Timisoara on May 7, 2014 and received the registration number 38/2014.

\section{Anthropometric, laboratory, and clinical assessments}

Data regarding patient's age and DM history - including the history of DM treatment - were collected from the patients' medical records. The $\mathrm{HbA}_{1 \mathrm{c}}$ level was measured using a National Glycohemoglobin Standardizatoin Program (NGSP)standardized and Diabetes Control and Complications Trial (DCCT)-compliant immune-turbidimetric assay (Hoffman-La Roche Ltd., Basel, Switzerland), having an inter-measurement coefficient of variation of $1.64 \%$ according to manufacturer's specifications. Lipid profile measurements were performed after at least 12 hours of fasting, and the blood drawn was analyzed using chemiluminescent standardized method. The Michigan Neuropathy Screening Instrument (MNSI) was used to diagnose $\mathrm{DN}$ and was performed by trained personnel. MNSI is a validated instrument for scoring DN, being widely used for the diagnosis and quantification of distal symmetrical peripheral neuropathy in diabetes. The MNSI includes two separate assessments: a 15 -item self-administered questionnaire that is scored by summing abnormal responses provided by the patient and a lower extremity examination that includes inspection and assessment of vibratory sensation and ankle reflexes, in which the abnormal findings are scored. The MNSI provides better sensitivity and specificity than the individual DN tests performed separately; the diagnosis of DN was considered positive in case of questionnaire score higher or equal to 7 or in case of a clinical examination score higher or equal to $2.5 .^{1,19}$

To evaluate the presence and the severity of depression, we used the Patient's Health Questionnaire-9 (PHQ-9). 
With its nine items, the PHQ-9 test consists of the actual nine criteria on which the diagnosis of DSM-IV depressive disorders is based. ${ }^{15}$ The PHQ-9 is a dual-purpose instrument which can establish, with the same nine items, depressive distress as well as grade depressive symptom severity. A higher PHQ-9 score is associated with more severe depression. Based on PHQ-9 score, the depression severity may be divided into three groups: minimal or mild (PHQ-9 score $<10$ ), moderate (PHQ-9 score 10-19), and severe (PHQ score $>19$ ).

The quality of DRSCA was evaluated using the Summary of Diabetes-Related Self Care Activities (SDSCA) questionnaire, which is a validated tool in the population of DM patients. A higher SDSCA score is associated with a better disease self-care in these patients. The SDSCA questionnaire includes items assessing the following aspects of the diabetes care: general and specific diet measures, exercise, blood glucose testing, foot care, and smoking. It was proven that the SDSCA questionnaire is a brief yet reliable and valid self-report measure of diabetes self-management, useful for both research and clinical practice. ${ }^{20}$

The diagnosis and severity grading of chronic kidney disease (CKD) were done according to the KDIGO 2012 guidelines. The positive diagnosis comprised either positive markers of kidney damage or decreased glomerular filtration rate, both present for a duration longer than 3 months. ${ }^{21}$ Positive diagnosis for diabetic retinopathy was established during a funduscopic exam, performed by the same ophthalmologist, trained and specialized in diagnosis of the diabetic lesions of the retina.

\section{Statistical analysis}

Data were collected and analyzed using the SPSS v17 software suite (SPSS Inc., Chicago, IL, USA) and are presented as mean \pm standard deviation (SD) for continuous variables with Gaussian distribution, median and interquartile range for continuous variables without Gaussian distribution, or percentages for categorical variables. To assess the significance of differences between the groups, Student's $t$-test or analysis of variance (ANOVA) (means, Gaussian populations), Mann-Whitney $U$-test or KruskalWallis (medians, non-Gaussian populations), and chi-square (proportions) tests were used. Continuous variable distributions were tested for normality using Shapiro-Wilk test, and for equality of variances using Levene's test.

The strength of the association between two continuous variables from non-Gaussian populations was evaluated using Spearman's correlation coefficient, and statistical significance was assessed using $t$-score distribution's test.
The individual impact of several confounding factors on the variance of a continuous variable was assessed by building multivariate regression models, and if the dependent variable was dichotomous by building multivariate logistic regressions. The predictors, in the final regression equations, were accepted according to a repeated backward stepwise algorithm (inclusion criteria: $P<0.05$, exclusion criteria: $P>0.10)$ in order to obtain the most appropriate theoretical model to fit the collected data. The quality of the model was described using the accuracy of prediction and also by adjusted $R^{2}$ (multivariate linear regression) and Nagelkerke's $R^{2}$ (logistic regression).

In this study, a $P$-value lower than 0.05 was considered the threshold for the statistical significance.

\section{Results \\ Studied group baseline characteristics}

The enrolled cohort included 80 men (43.5\%) and 104 women $(56.5 \%)$, having a median age of 64 years (range: 52 to 86 years) and a median diabetes duration of 7 years (range: 0 to 28 years). The prevalence of diabetes complications and comorbidities was in the expected ranges for the type of studied population: $28.8 \%$ (53) had DN, 25.5\% (47) had CKD, 32.1\% (59) had retinopathy, and $82.6 \%$ of them (152 individuals) had hypertension. The detailed baseline characteristics are presented in Table 1.

\section{Depression prevalence}

In our study group, $53.3 \%$ of the patients had moderate depression, 17.9\% had severe depression, and 28.8\% had no depression symptoms. While the differences regarding the prevalence of absent and moderate depression were not significant between the sexes, female sex was associated with an increased prevalence of severe depression $(21.2 \%$ vs $13.8 \% ; P=0.026)$.

Table I Studied group baseline characteristics

\begin{tabular}{|c|c|}
\hline Male sex $(\%)^{\mathrm{a}}$ & $80(43.5 \%)$ \\
\hline Age $\left(\right.$ years) ${ }^{\mathrm{b}}$ & $64(11)$ \\
\hline Diabetes duration $^{\mathrm{b}}$ & $7(8)$ \\
\hline $\mathrm{HbA}_{\mathrm{lc}}(\%)^{\mathrm{c}}$ & $8.14 \pm 1.70$ \\
\hline BMI $\left(\mathrm{kg} / \mathrm{m}^{2}\right)^{\mathrm{c}}$ & $30.7 \pm 4.3$ \\
\hline Neuropathy (\%) ${ }^{\mathrm{a}}$ & $53(28.8 \%)$ \\
\hline Chronic kidney disease (\%) & $47(25.5 \%)$ \\
\hline Diabetic retinopathy (\%) & $59(32.1 \%)$ \\
\hline Hypertension (\%) & 152 (82.6\%) \\
\hline
\end{tabular}

Notes: aDichotomous variables. Results are presented as number of individuals and (percentage from total). 'Numerical variables without Gaussian distribution. Results are presented as median (interquartile range). 'Numerical variables with Gaussian distribution. Results are presented as mean \pm standard deviation.

Abbreviation: BMI, body mass index. 
In patients with T2DM, increase in depression severity was associated with higher age, longer diabetes duration, increased BMI, and increased prevalence of T2DM chronic complications (CKD, retinopathy, and neuropathy severity). The results of the comparison are presented in Table 2.

It is worth mentioning that, despite nonsignificant variance between the three analyzed sub-groups, at the post hoc analysis (Bonferroni) we observed significant differences with regard to BMI and increase in waist circumference between the subgroups having severe vs absent or moderate depression.

\section{Development of depression in patients with T2DM}

To evaluate the impact of different predictors in the development of depression in patients with T2DM, we created a multivariate logistic regression model having as outcome the presence of depression and as initial predictors: patient's age, diabetes duration, the neuropathy severity score (MNSI), the cross-sectional value of $\mathrm{HbA}_{1 \mathrm{c}}$, BMI, and the presence of $\mathrm{CKD}$ and diabetic retinopathy. To find the best model that suits this relationship, the final regression equation was validated according to a backward stepwise algorithm, having an inclusion threshold criterion for predictors a $P$-value for goodness of fit lower or equal to 0.05 respectively an exclusion threshold a $P$-value higher than 0.10 . In the final regression equation, the following predictors were accepted: age, neuropathy severity, BMI value, and value of $\mathrm{HbA}_{1 \mathrm{c}}$.

According to our model, the incidence of depression was significantly associated with higher age, more severe neuropathy symptoms, and an elevated BMI. The crosssectional value of $\mathrm{HbA}_{1 \mathrm{c}}$ was only a marginally significant predictor for depression development. For increase of 1 year in patient's age, we observed an increase of $4.8 \%$ in the odds for developing depression (odds ratio [OR] $=1.048$; $P=0.024$ ); for each increase of one point in the MNSI score, we observed an increase of $45.6 \%$ in the odds of developing depression $(\mathrm{OR}=1.456 ; P<0.001)$; for each increase in $\mathrm{BMI}$ of $1 \mathrm{~kg} / \mathrm{m}^{2}$, we observed an increase of $10.1 \%$ in the odds of developing depression ( $\mathrm{OR}=1.101 ; P=0.037)$. The impact of significant predictors on the development of depression is presented in Table 3 .

\section{Depression influences the DRSCA}

At the time of comparison of DRSCA interventions in our study cohort, stratified according to the presence and severity of depression, we observed that more severe depression was associated with a significant decrease in the SDSCA global score ( 24 vs 33 vs 37 points; $P<0.001$; Figure 1), in the diet interventions score ( 12 vs 16 vs 18 points; $P<0.001$ ), physical exercise interventions ( 5 vs 6 vs 8 points; $P<0.001$ ), and feet care score ( 4 vs 5 vs 7 points; $P=0.017$ ). The differences regarding the quality of glycemic self-monitoring were not significantly influenced by the presence or severity of depression (Table 4).

The value of the PHQ-9 score was reverse correlated with the SDSCA global score and all the subcomponents of the score, thus revealing that a more severe depression is associated with decreases in the quality of the DRSCA. Except for the self-monitoring blood glucose score, all the

Table 2 The impact of depression severity on the studied parameters

\begin{tabular}{|c|c|c|c|c|}
\hline & $\begin{array}{l}\text { No depression } \\
(n=53)\end{array}$ & $\begin{array}{l}\text { Moderate depression } \\
(\mathrm{n}=98)\end{array}$ & $\begin{array}{l}\text { Severe depression } \\
(n=33)\end{array}$ & $P$-value \\
\hline Age (years) ${ }^{\mathrm{a}}$ & $62(13)$ & $65(10)$ & $64(13)$ & 0.395 \\
\hline Diabetes duration (years) ${ }^{\mathrm{a}}$ & $6(8)$ & $7(7)$ & $7(10)$ & 0.675 \\
\hline MNSI (score) ${ }^{\mathrm{a}}$ & $3(3)$ & $5(2)$ & $7(4)$ & $<0.00 I^{*}$ \\
\hline CKD prevalence ${ }^{b}$ & $13.2 \%(7)$ & $23.5 \%(23)$ & $51.5 \%(17)$ & $<0.00 I^{*}$ \\
\hline Retinopathy prevalence ${ }^{b}$ & $20.8 \%(I I)$ & $29.6 \%(29)$ & $57.6 \%(19)$ & $0.002 *$ \\
\hline $\mathrm{HbA}_{\mathrm{Ic}}(\%)^{\mathrm{c}}$ & $7.8 \pm 2.1$ & $8.2 \pm 1.4$ & $8.7 \pm 1.8$ & $0.042 *$ \\
\hline BMI $\left(\mathrm{kg} / \mathrm{m}^{2}\right)^{\mathrm{c}}$ & $30.7 \pm 4.4$ & $30.2 \pm 4.4$ & $31.8 \pm 3.4$ & 0.164 \\
\hline Exceed in waist circumference $(\mathrm{cm})^{c}$ & $20.6 \pm 17.3$ & $18.5 \pm 17.2$ & $24.9 \pm 13.5$ & 0.243 \\
\hline Total cholesterol $(\mathrm{mg} / \mathrm{dL})^{c}$ & $214.4 \pm 62.2$ & $199.5 \pm 53.5$ & $196.8 \pm 61.4$ & 0.248 \\
\hline LDL cholesterol $(\mathrm{mg} / \mathrm{dL})^{\mathrm{c}}$ & $130.4 \pm 52 . \mid$ & $120.6 \pm 43.2$ & $119.8 \pm 42.5$ & 0.139 \\
\hline $\mathrm{HDL}$ cholesterol $(\mathrm{mg} / \mathrm{dL})^{\mathrm{c}}$ & $46.0 \pm 9.4$ & $46.4 \pm 10.5$ & $42.2 \pm 12.4$ & 0.410 \\
\hline Triglycerides $(\mathrm{mg} / \mathrm{dL})^{c}$ & $197.3 \pm 86.0$ & $161.6 \pm 77.0$ & $161.2 \pm 68.4$ & $0.021^{*}$ \\
\hline
\end{tabular}

Notes: *Differences are significant at $\alpha=0.05$ threshold. a Numerical variables without Gaussian distribution. Results are presented as median (interquartile range). $P$-value was calculated using Kruskal-Wallis test. ${ }^{b}$ Results are presented as percentage from total (number of individuals). P-value was calculated using chi-square (trend) test. ${ }^{C}$ Numerical variables with Gaussian distribution. Results are presented as mean \pm standard deviation. $P$-value was calculated using one-way ANOVA test.

Abbreviations: MNSI, Michigan Neuropathy Screening Instrument; CKD, chronic kidney disease; BMI, body mass index; LDL, low-density lipoprotein; HDL, high-density lipoprotein; ANOVA, analysis of variance. 
Table 3 Multivariate logistic regression analysis parameters (dependent variable - the presence of depression)

\begin{tabular}{|c|c|c|c|c|c|}
\hline Predictor & B & Wald & $\operatorname{Exp}(\beta)$ & $95 \% \mathrm{Cl}(\operatorname{Exp}[\beta])$ & $P$-value \\
\hline Patient's age (per I year increase) & 0.243 & 2.162 & 1.048 & (I.0II to 1.085$)$ & 0.024 \\
\hline MNSI score (per one point increase) & 0.385 & 15.842 & 1.456 & (1.236 to 1.716$)$ & $<0.001$ \\
\hline BMI (per one $\mathrm{kg} / \mathrm{m}^{2}$ increase) & 0.182 & 1.488 & 1.101 & (1.035 to 1.166$)$ & 0.037 \\
\hline $\mathrm{HbA}_{\mathrm{Ic}}$ (per one percentage point increase) & 0.104 & 0.855 & 1.240 & (0.886 to 1.594$)$ & 0.086 \\
\hline
\end{tabular}

Abbreviations: MNSI, Michigan Neuropathy Screening Instrument; BMI, body mass index; Cl, confidence interval.

studied associations had a negative Spearman's correlation coefficient (Table 5), the correlations being statistically significant.

According to our models, the variation of the PHQ-9 score explained 9\% of the SDSCA's global score variation. The association between these two components is a negative, moderate, and significant one (Spearman's $r=-0.305$; $P<0.001$; Figure 2).

Regarding the other studied subcomponents of DRSCA, we observed negative, moderate, and significant correlations with the severity of depression for the following components: diet interventions (Spearman's $r=-0.297 ; P<0.001$ ) and physical exercise interventions (Spearman's $r=-0.388$; $P<0.001)$. The correlation between the severity of depression and feet care quality was a negative, weak, but statistically significant one (Spearman's $r=-0.180 ; P=0.015$ ). The associations between these components are presented in Figure 3.

\section{Depression: more than a confounding factor regarding diabetes self-care}

To evaluate the impact of depression on the quality of DRSCA as an independent predictor, to overcome the possible biases in the relationship like patient's age, sex, or BMI,

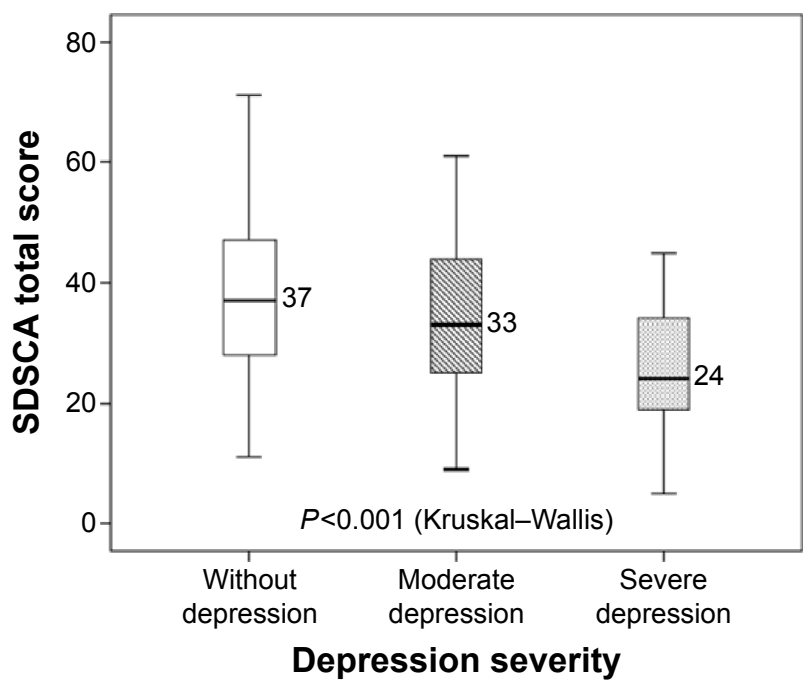

Figure I Comparison of SDSCA total score according to depression severity. Abbreviation: SDSCA, Summary of Diabetes-Related Self Care Activities. we created a multivariable regression having the SDSCA total score as a dependent variable and the depression score according to PHQ-9 questionnaire as the independent variable. The relationship was adjusted for age, diabetes duration, sex, and the presence of diabetes complications (neuropathy, CKD, and retinopathy). The obtained model revealed a significant impact of depression on the quality of self-care even after the adjustment for the other covariables; for increase of one SD in the PHQ-9 score, we observed a decrease of 0.212 SDs in the total SDSCA score $(\mathrm{B}=-0.313 ; \beta=-0.212 ; P=0.004)$. We can also conclude, from the model built, that the following predictors act in an independent manner with respect to the SDSCA score: patient's age $(B=-0.282 ; \beta=-0.183, P=0.014)$, presence of $\mathrm{DN}(\mathrm{B}=-5.528 ; \beta=-0.189 ; P=0.016)$, and presence of $\mathrm{CKD}$ $(\mathrm{B}=-6.994 ; \beta=-0.230 ; P=0.003)$.

\section{Discussion}

The results of our study point to an increased prevalence of depression in patients with T2DM, which can be explained on one hand by the negative impact of the presence of a chronic, incurable disease on the mental state of the patient and on the other hand by the fact that both depression and T2DM have a partial common pathologic background. ${ }^{22}$ The vast majority of the studied patients, however, presented mild or moderate depression $(53.3 \%){ }^{23}$

From the literature search that we performed, this is the only study in the Western part of Romania that assessed the relationship between the presence of depression, a frequent condition to be found in elderly patients, a statement valid not only for the general population but also for patients with T2DM. In patients with T2DM, beyond the common consequences of depression commonly observed in general population, the presence of this condition may have a serious negative impact on the global health and well-being of the patients, especially on those components which are related to the self-management of diabetes, an aspect of paramount importance since it is known that DM is a chronic, lifelong disease for which management should be performed mainly by the patient. 
Table 4 The impact of depression severity on the quality of diabetes-related self-care activities

\begin{tabular}{|c|c|c|c|c|}
\hline & $\begin{array}{l}\text { No depression } \\
(n=53)\end{array}$ & $\begin{array}{l}\text { Moderate depression } \\
(\mathrm{n}=98)\end{array}$ & $\begin{array}{l}\text { Severe depression } \\
(n=33)\end{array}$ & $P$-value \\
\hline SDSCA global score & $37(20)$ & $33(19)$ & $24(16)$ & $<0.00 I^{*}$ \\
\hline SDSCA diet intervention score & $18(9)$ & $16(7)$ & $12(7)$ & $<0.00 I^{*}$ \\
\hline SDSCA exercise score & $8(5)$ & $6(4)$ & $5(5)$ & $<0.00 I^{*}$ \\
\hline SDSCA glycemic monitoring score & $5(13)$ & $7(11)$ & $4(8)$ & 0.394 \\
\hline SDSCA feet care score & $7(8)$ & $5(7)$ & $4(4)$ & $0.017^{*}$ \\
\hline
\end{tabular}

Notes: *Differences are significant at $\alpha=0.05$ threshold. Results are presented as median (interquartile range). P-value was calculated using Kruskal-Wallis test.

Abbreviation: SDSCA, Summary of Diabetes-Related Self Care Activities.

In our study group, $53.3 \%$ of the patients had moderate depression, $17.9 \%$ had severe depression, and $28.8 \%$ had no depression symptoms. While the differences regarding the prevalence or absence of moderate depression were not significant between the sexes, female sex was associated with an increased prevalence of severe depression $(21.2 \%$ vs $13.8 \% ; P=0.026$ ).

Similar studies (systematic reviews) depict high rates of comorbidity for depression and diabetes. ${ }^{6,9}$ The prevalence rate of depression is more than three times higher in people with $\operatorname{T} 1 \mathrm{DM}(12 \%$, range $5.8 \%-43.3 \%$ vs $3.2 \%$, range $2.7 \%-11.4 \%$ ) and nearly twice as high in people with T2DM (19.1\%, range $6.5 \%-33 \%$ vs $10.7 \%$, range $3.8 \%-19.4 \%)$ compared to those without. Women with diabetes and also women without diabetes experience a higher prevalence of depression than men. ${ }^{9}$

In another review of the literature, the prevalence of comorbid depression was significantly higher in diabetic women $(28 \%)$ than in diabetic men $(18 \%)$, in uncontrolled $(30 \%)$ than in controlled (21\%) studies, in clinical $(32 \%)$ than in community (20\%) samples, and when assessed by self-report questionnaires (31\%) than by standardized diagnostic interviews $(11 \%) .{ }^{6} \mathrm{We}$ observed that a more severe depression, quantified by an increased PHQ-9 score, had a negative impact on the quality of diabetes-related self-management of the disease, which may act in the form of a loop, ie, by decreasing the quality of diabetes-related

Table 5 Correlation between depression score and SDSCA scores

\begin{tabular}{lll}
\hline & PHQ-9 score & P-value \\
\hline SDSCA global score & -0.305 & $<0.00 I^{*}$ \\
SDSCA diet intervention score & -0.297 & $<0.00 I^{*}$ \\
SDSCA exercise score & -0.388 & $<0.00 I^{*}$ \\
SDSCA glycemic monitoring score & -0.055 & 0.455 \\
SDSCA feet care score & -0.180 & $0.015^{*}$ \\
\hline
\end{tabular}

Notes: *Correlations are significant at $\alpha=0.05$ threshold. Results are presented as Spearman's rank sum correlation coefficient. $P$-value was calculated using $t$-value distribution test.

Abbreviations: PHQ-9, Patient's Health Questionnaire-9; SDSCA, Summary of Diabetes-Related Self Care Activities. self-care, depression may lead to the worsening of glycemic control, increase of depression severity, and so on. ${ }^{8}$ Depression has been proved to have a negative impact on diet interventions, exercise practice, and feet care, but not on the quality of glycemic self-monitoring., ${ }^{42}$ This may be explained by the fact that the intensity of glycemic self-monitoring is usually a task designated by the physician and thus in many patients may be an adopted standard of care.

An interesting observation is that depression not only acts as a confounding factor but also acts in an independent manner on the quality of self-care. ${ }^{12}$ If we would not have performed this type of demonstration, one might think that the study's observation may be a consequence of a longer diabetes duration, higher age, or other cofactors, which are also associated with the presence of depression.

The interventions of the health care professionals can take place only periodically and in cases in which a prompt measure is needed (eg, the acute complications of DM), however the disease needs continuous intervention from the patient, usually actions needing to be performed many times. Thus, we can conclude that our article addresses a usually

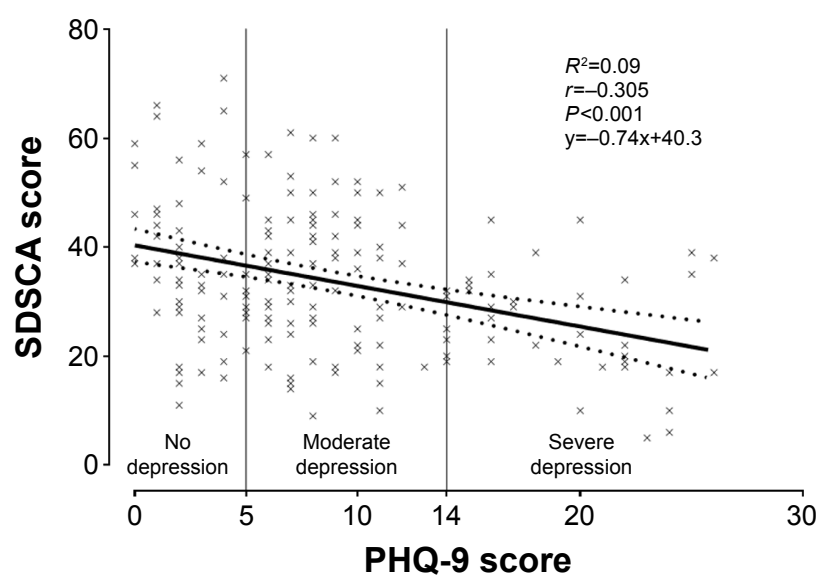

Figure 2 Association between depression and quality of diabetes-related self-care activities.

Abbreviations: SDSCA, Summary of Diabetes-Related Self Care Activities; PHQ-9, Patient's Health Questionnaire-9. 

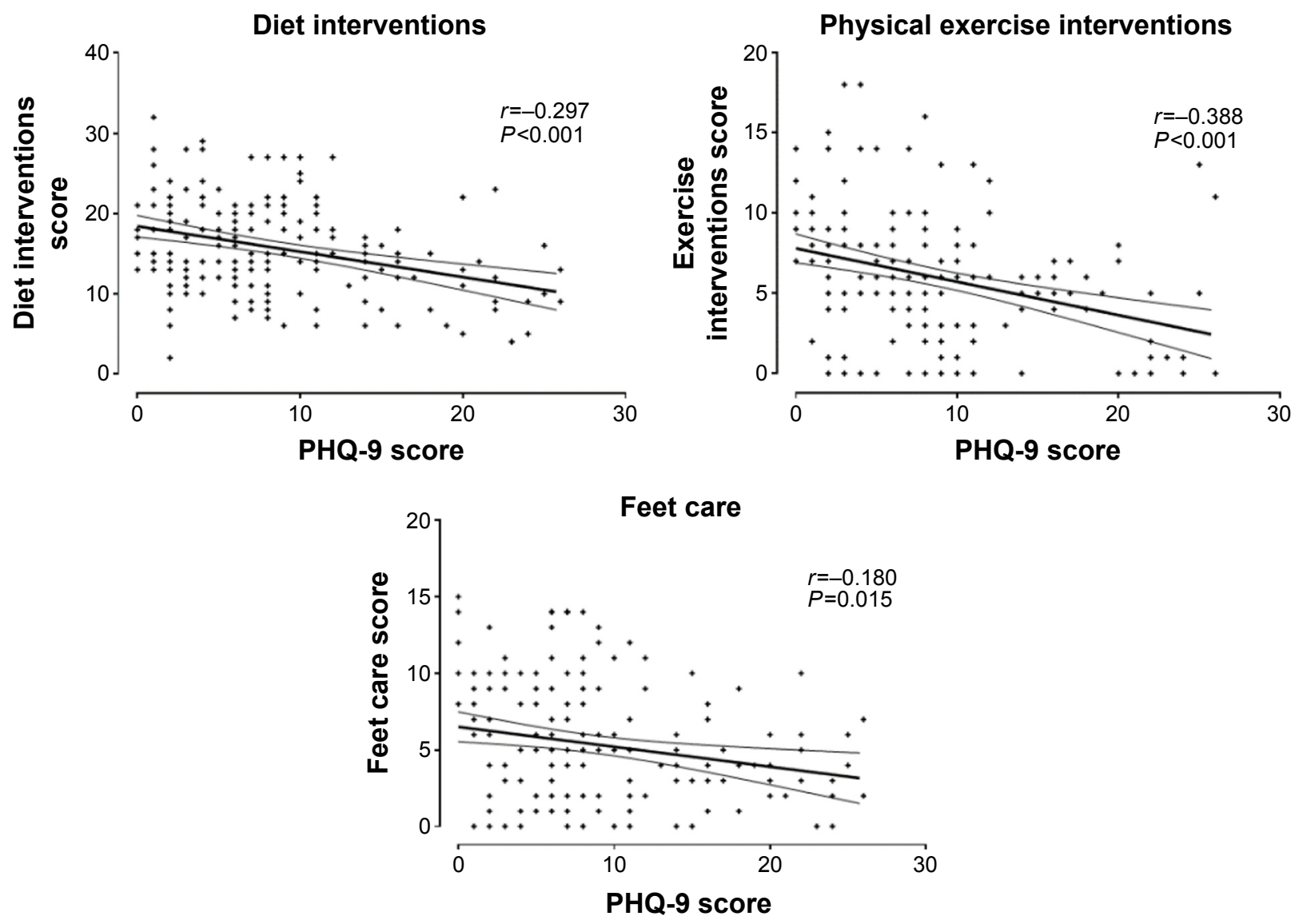

Figure 3 Association between depression severity and the subcomponents of diabetes-related self-care activities. Abbreviation: PHQ-9, Patient's Health Questionnaire-9.

neglected component of T2DM, the presence of depression, its consequences, and its mediated impact on the quality of overall diabetes management.

The main issue we identified in relation to our study is that the tool used for diagnosing the depression, the PHQ-9 questionnaire, has a series of limitations regarding its performance in the detection of depression. However, as stated in literature, there is no golden standard test to be used for depression diagnosis that is feasible in outpatient setting. In our example, we used the PHQ-9 test as a screening method, since it has a high sensitivity with an increased negative predictive value which makes it an appropriate screening method. In case of a positive diagnosis, we followed up the patient and further investigations were performed to establish the certain diagnosis. However, the cross-sectional design of this study may have a series of limitations, mainly due to the long time needed for the predictors analyzed to act before leading to the development of diabetic complications such as neuropathy, $\mathrm{CKD}$, retinopathy, or macrovascular diseases. The tools used to evaluate the quality of glycemic control in a cross-sectional setting reflect the values of only the previous
3 months (eg, $\mathrm{HbA}_{1 \mathrm{c}}$ value), and since longer time is needed for the development of diabetes complications, this may lead to biases in the interpretation of this association. However, we started from the premise that it is known that in patients with T2DM, even the cross-sectional measurement of $\mathrm{HbA}_{10}$ value might be a valuable tool in evaluating the long-term metabolic balance, the changes being not so important when compared to patients with T1DM.

We considered, based on the logic of the relationship between the studied items, that these two associations are part of a larger circle of associations, and have an important impact on the overall management of diabetes as follows: first, the incidence of depression may have a series of particularities in patients with diabetes, compared to general population. In addition, the factors leading to the onset of depression may be very different compared to the general population (even if we just have in mind the impact of having a chronic, life-lasting disease, we might change completely our point of view over depression). On the other hand, the presence of depression may influence the patient's attention and interest on the management of the disease, leading thus 
to impairment of the quality of DRSCA. This impairment will further lead to the deterioration of glycemic control, thus augmenting a series of factors which may emphasize the depression's magnitude.

When we designed this study, we expected an increased prevalence of depression in our cohort of patients; however, the results of the study demonstrated that even our expectation underestimated the real magnitude of this health issue.

The findings of our study demonstrate an increased prevalence of depression and its consequences, an underestimated condition in patients with T2DM. The overall impact on patient's health and on diabetes management stresses the importance of screening for depression in this patient population, and in the case of a positive diagnosis, on the importance of a firm and adequate treatment. These statements are emphasized by the feasibility of performing this type of depression screening test and by the fact that prompt intervention in the correction of depression symptoms may have a paramount importance in both quality of life and quality of overall diabetes management.

\section{Conclusion}

Screening for depression should be part of the standard of care in patients with T2DM. The prevalence of depression is significantly higher in patients with T2DM compared to general population. Depression has a major negative impact on the overall health state of the patients with T2DM not only in a direct manner but also indirectly, mediated by the consecutive decreases in the quality of self-management of the disease.

\section{Disclosure}

The authors report no conflicts of interest in this work.

\section{References}

1. American Diabetes Association. Standards of Medical Care in Diabetes - 2015. Diabetes Care. 2015;38(Suppl 1):S1-S93.

2. Marrero DG, Ard J, Delamater AM, et al. Twenty-first century behavioral medicine: a context for empowering clinicians and patients with diabetes: a consensus report. Diabetes Care. 2013;36:463-470.

3. Norris SL, Lau J, Smith SJ, Schmid CH, Engelgau MM. Self-management education for adults with type 2 diabetes: a meta-analysis of the effect on glycemic control. Diabetes Care. 2002;25:1159-1171.

4. Anderson RJ, Grigsby AB, Freedland KE, et al. Anxiety and poor glycemic control: a meta-analytic review of the literature. Int J Psychiatry Med. 2002;32:235-247.

5. Delahanty LM, Grant RW, Wittenberg E, et al. Association of diabetesrelated emotional distress with diabetes treatment in primary care patients with type 2 diabetes. Diabet Med. 2007;24:48-54.
6. Anderson RJ, Freedland KE, Clouse RE, Lustman PJ. The prevalence of comorbid depression in adults with diabetes: a meta-analysis. Diabetes Care. 2001;24:1069-1078.

7. Kovacs Burns K, Nicolucci A, Holt RIG, et al; DAWN2 Study Group. Diabetes Attitudes, Wishes and Needs second study (DAWN2Ô): cross-national benchmarking indicators for family members living with people with diabetes. Diabet Med. 2013;30:778-788.

8. Fisher EB, Chan JC, Nan H, Sartorius N, Oldenburg B. Co-occurrence of diabetes and depression: conceptual consideration for an emerging global health challenge. J Affect Disord. 2012;142(Suppl 1):S56-S66.

9. Roy T, Lloyd CE. Epidemiology of depression and diabetes: a systematic review. J Affect Disord. 2012;142(Suppl 1):S8-S21.

10. Grigsby AB, Anderson RJ, Freedland KE, Clouse RE, Lustman PJ. Prevalence of anxiety in adults with diabetes: a systematic review. J Psychosom Res. 2002;53:1053-1060.

11. Hermanns N, Kulzer B, Krichbaum M, Kubiak T, Haak T. Affective and anxiety disorders in a German sample of diabetic patients: prevalence, comorbidity and risk factors. Diabet Med. 2005;22:293-300.

12. Schmitz N, Gariépy G, Smith KJ, et al. Recurrent subthreshold depression in type 2 diabetes: an important risk factor for poor health outcomes. Diabetes Care. 2014;37:970-978.

13. Katon W, Fan MY, Unützer J, Taylor J, Pincus H, Schoenbaum M. Depression and diabetes: a potentially lethal combination. J Gen Intern Med. 2008;23:1571-1575.

14. Tabák AG, Akbaraly TN, Batty GD, Kivimäki M. Depression and type 2 diabetes: a causal association? Lancet Diabetes Endocrinol. 2014;2:236-245.

15. Kroenke K, Spitzer RL, Williams JBW. The PHQ-9: validity of a brief depression severity measure. J Gen Intern Med. 2001;16:606-613.

16. Strandberg RB, Graue M, Wentzel-Larsen T, Peyrot M, Rokne B. Relationship of diabetes-specific emotional distress, depression, anxiety, and overall well-being with $\mathrm{HbA} 1 \mathrm{c}$ in adult person with type 1 diabetes. J Psychosom Res. 2014;77:174-179.

17. Fisher L, Mullan JT, Arean P, Glasgow RE, Hessler D, Masharani U. Diabetes distress but not clinical depression and depressive symptoms is associated with glycemic control in both cross-sectional and longitudinal analyses. Diabetes care. 2010;33:23-28.

18. Fisher L, Skaff MM, Mullan JT, et al. Clinical depression versus distress among patients with type 2 diabetes: not just a question of semantics. Diabetes care. 2007;30:542-548.

19. Herman WH, Pop-Busui R, Braffett BH, et al; DCCT/EDIC Research Group. Use of the Michigan Neuropathy Screening Instrument as a measure of distal symmetrical peripheral neuropathy in type 1 diabetes: results from the Diabetes Control and Complications Trial/ Epidemiology of Diabetes Interventions and Complications. Diabet Med. 2012;29:937-944.

20. Toobert DJ, Hampson SE, Glasgow RE. The summary of diabetes self-care activities measure. Diabetes Care. 2000;23:943-950.

21. Kidney Disease: Improving Global Outcomes (KDIGO) CKD Work Group. KDIGO 2012 Clinical Practice Guideline for the Evaluation and Management of Chronic Kidney Disease. Kidney inter. 2013; 86(Suppl 3):1-150.

22. Starkstein SE, Davis WA, Dragovic M, Cetrullo V, Davis TME, Bruce DG. Diagnostic criteria for depression in type 2 diabetes: a datadriven approach. PLoS One. 2014;9(11):e112049.

23. Bruce DG, Davis WA, Starkstein SE, Davis TM. A prospective study of depression and mortality in patients with type 2 diabetes: The Fremantle Diabetes Study. Diabetologia. 2005;48(12):2532-2539. 
Clinical Interventions in Aging

\section{Publish your work in this journal}

Clinical Interventions in Aging is an international, peer-reviewed journal focusing on evidence-based reports on the value or lack thereof of treatments intended to prevent or delay the onset of maladaptive correlates of aging in human beings. This journal is indexed on PubMed Central, MedLine,

CAS, Scopus and the Elsevier Bibliographic databases. The manuscript management system is completely online and includes a very quick and fair peer-review system, which is all easy to use. Visit http://www.dovepress. $\mathrm{com} /$ testimonials.php to read real quotes from published authors.

Submit your manuscript here: http://www.dovepress.com/clinical-interventions-in-aging-journal 